\title{
Der Orthopäde
}

\section{Einführung zum Thema}

Orthopäde $2019 \cdot 48: 451$

https://doi.org/10.1007/s00132-019-03757-y

(c) Springer Medizin Verlag GmbH, ein Teil von Springer Nature 2019

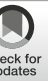

\section{Michael Akbar ${ }^{1} \cdot$ Thomas Dreher $^{2} \cdot$ Ralf Stü $_{\text {cker }}^{3}$}

'Zentrum für Orthopädie, Unfallchirurgie und Paraplegiologie, Klinik für Orthopädie und Unfallchirurgie, Universitätsklinik Heidelberg, Heidelberg, Deutschland

${ }^{2}$ Universitäts-Kinderspital Zürich - Eleonorenstiftung, Zürich, Schweiz

${ }^{3}$ Altonaer Kinderkrankenhaus Hamburg und Universitätsklinik Hamburg Eppendorf, Hamburg, Deutschland

\section{Wachstumsbedingte Störung der Halte- und Bewegungsorgane}

Ihr

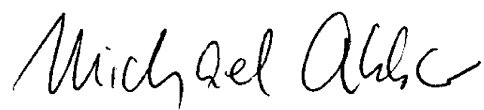

PD Dr. Michael Akbar

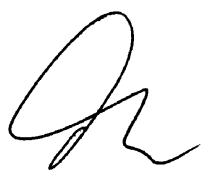

Professor Dr. Thomas Dreher
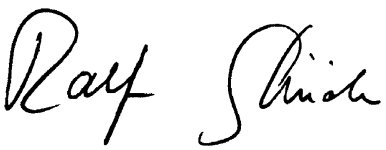

Professor Dr. Ralf Stücker
Korrespondenzadresse

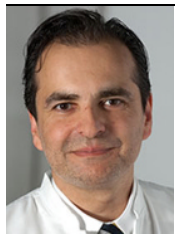

PD Dr. Michael Akbar

Zentrum für Orthopädie, Unfallchirurgie und Paraplegiologie, Klinik für Orthopädie und Unfallchirurgie,

Universitätsklinik Heidelberg

Schlierbacher Landstr.

200a, 69118 Heidelberg,

Deutschland

Michael.akbar@

med.uni-heidelberg.de

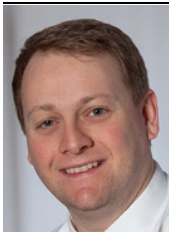

Professor Dr. Thomas Dreher Universitäts-Kinderspital Zürich - Eleonorenstiftung Steinwiesstrasse 75, 8032 Zürich, Schweiz thomas.dreher@kispi.uzh.ch der Halte- und Bewegungsorgane sowie der Wirbelsäule. Das Wissen um die Entwicklungs- und Wachstumsphasen des Kindes und das frühzeitige Erkennen von Wachstums- und Entwicklungsstörungen, Fehlbildungen sowie Anomalien und Normabweichungen ermöglichen uns als Orthopäden und Kinderorthopäden, einen positiven Einfluss auf die Entwicklung des noch wachsenden $\mathrm{Pa}$ tienten zu nehmen. Wir können damit den frühzeitigen Gelenkverschleiß, unphysiologische Bewegungsabläufe und schmerzhafte Haltungs- und Bewegungsstörungen im Erwachsenenalter versuchen $\mathrm{zu}$ verhindern.

Wir wünschen Ihnen viel Freude bei der Durchsicht der folgenden Beiträge und bedanken uns bei allen Autoren, die dieses Heft ermöglicht haben. 